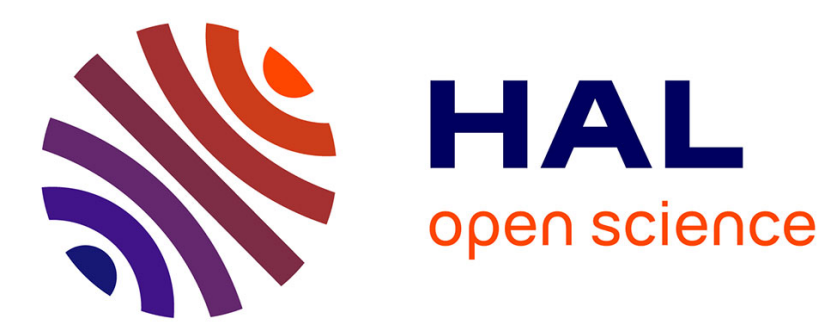

\title{
Hauriou et la théorie de l'institution
}

Éric Millard

\section{To cite this version:}

Éric Millard. Hauriou et la théorie de l'institution. Droit et Société, 1995, 30/31, pp.381-412. halshs00125759

\section{HAL Id: halshs-00125759 \\ https://shs.hal.science/halshs-00125759}

Submitted on 22 Jan 2007

HAL is a multi-disciplinary open access archive for the deposit and dissemination of scientific research documents, whether they are published or not. The documents may come from teaching and research institutions in France or abroad, or from public or private research centers.
L'archive ouverte pluridisciplinaire HAL, est destinée au dépôt et à la diffusion de documents scientifiques de niveau recherche, publiés ou non, émanant des établissements d'enseignement et de recherche français ou étrangers, des laboratoires publics ou privés. 


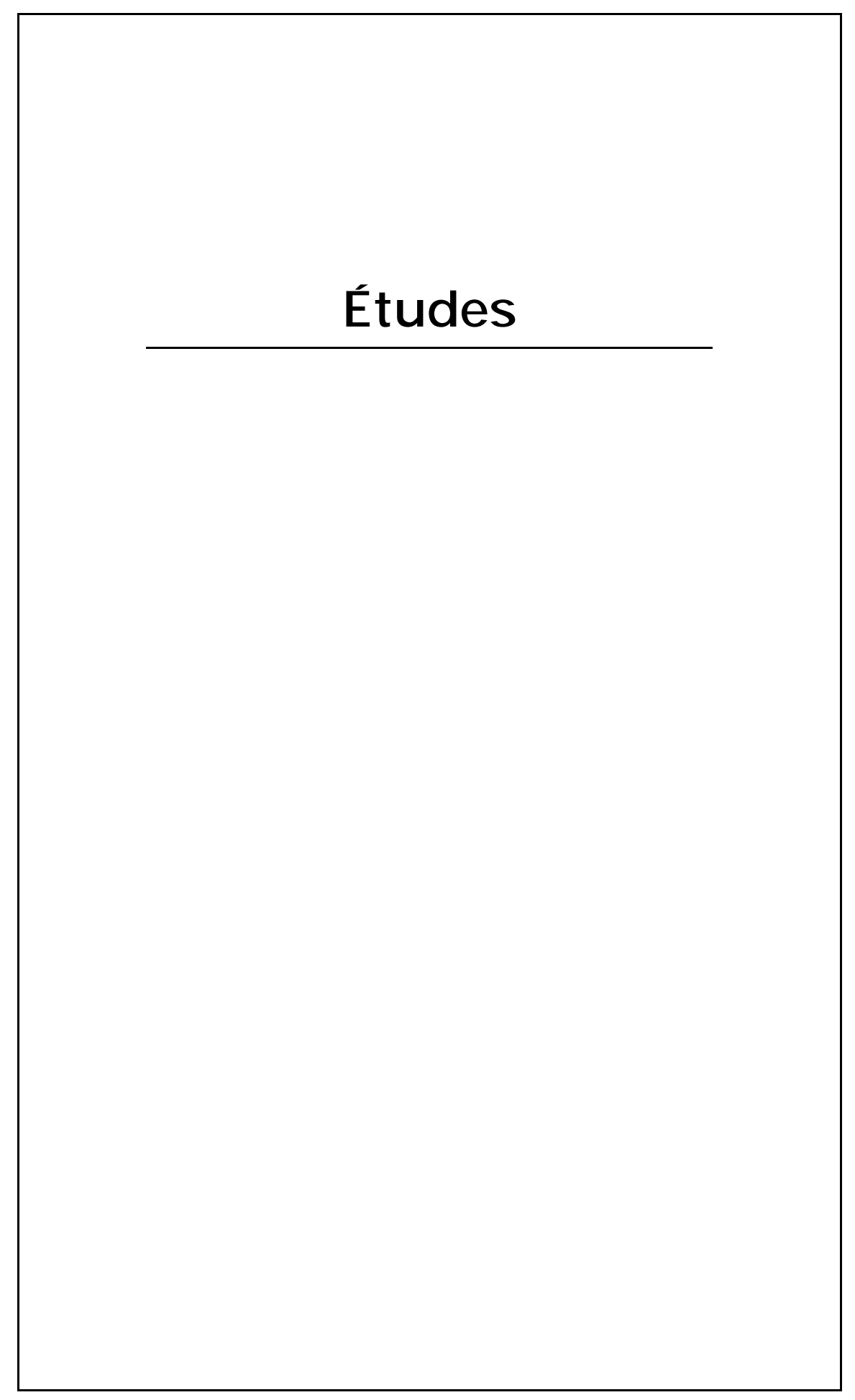





\section{et la théorie de l'institution *}

\section{Eric Millard **}

\section{Résumé}

La théorie de l'institution formulée par le doyen Maurice Hauriou au début du siècle demeure particulièrement stimulante pour une analyse théorique du droit et pour une compréhension de sa dimension sociale. L'article revient sur cette théorie. Après en avoir présenté la genèse et apprécié le contenu, l'auteur retrace sa postérité dans la doctrine juridique. Puis, à partir d'une évaluation critique de l'idéalisme institutionnel, et en tenant compte de l'apport des analyses institutionnelles en dehors de la sphère des juristes, il montre l'intérêt que revêt pour ceux-ci une réflexion sur la théorie proposée par Hauriou et son utilisation critique.

Analyse institutionnelle - Doctrine juridique - Épistémologie - Théorie de l'État - Théorie du droit.

\section{Summary}

Hauriou and the théorie de l'institution

The institution theory developed by dean Maurice Hauriou at the beginning of the century still remains especially stimulating for a theoretical analysis of law and for understanding its social scope. The article discusses this theory. After explaining its genesis and evaluating its content, the author retraces its ramifications in juridical doctrine. Then, based on a critical evaluation of institutional idealism, and taking into account the contribution of institutional analyses outside the realm of jurists, the author demonstrates the interest the theory proposed by Hauriou and its critical application may have for jurists.

Epistemology - Institutional analysis - Juridical doctrine - Theory of law Theory of the State.

\section{L'auteur}

Maître de conférences de droit public à l'Université de Toulouse Le Mirail, membre du Centre de recherches critiques sur le droit (CERCRID, URA CNRS 1155/

Université J ean Monnet de SaintEtienne) et du Laboratoire

FRAMESPA (France méridionale et Espagne: histoire des sociétés du Moyen Age à l'époque contemporaine, URA CNRS 247/Université de Toulouse Le Mirail). Participe aux travaux du

Centre de recherches sur

l'administration publique de Saint-Etienne (CERAPSE, Université J ean Monnet de SaintEtienne) et du Laboratoire de théorie du droit (Université J ean Moulin Lyon 3).

A collaboré à l'ouvrage publié sous la direction de M.-A. Cohendet : Droit public. Méthodes de travail (Paris, Montchrestien, 1994) et à celui du Centre d'études et de recherches d'histoire du droit : Religions, Eglises et Droit (Université de Saint-Etienne, 1990). Sa thèse de doctorat, Famille et droit public, est publiée à la LGDJ (coll.

«Bibliothèque de droit public », 1995).

* Cet article est tiré d'une intervention faite au cours d'un séminaire du CERCRID (Centre de minaire du CERChID (Centre de Université Jean Monnet de SaintEtienne) consacré à l'institution et qui s'est tenu à Goutelas-enForez le 20 mai 1995.

** FRAMESPA, URA CNRS 247, Maison de la Recherche,

Université de Toulouse Le Mirail, 5 , allées Antonio-Machado, F-31058 Toulouse cedex. 
E. Millard

Hauriou et la théorie de l'institution
L'institution : le mot est unique, les réalités qu'il désigne sont multiples. La théorie de l'institution de Maurice Hauriou n'est certes pas la première analyse de type institutionnel à avoir été proposée (Tanguy, 1991) ; elle représente pourtant dans la thématique des juristes, en France tout au moins, une étape essentielle de la conceptualisation de l'institution et de la théorie du droit : il n'est alors peut-être pas inutile de revenir sur les écrits du doyen toulousain pour tenter, sinon de comprendre de manière univoque le concept, du moins d'essayer de le situer dans le champ de la réflexion sur le droit.

L'effort est nécessaire : le terme institution revient en effet relativement fréquemment dans le discours des juristes, sans que I'on sache véritablement dans quel sens, par rapport à quelle analyse, et dans quel but il est employé. Cela ne peut qu'encourager un sentiment de méfiance à son égard car comme le note Jacques Chevallier : «Le concept d'institution est perçu comme trop statique alors que l'analyse institutionnelle recouvre des produits disparates et hétérogènes » (Chevallier, 1981). Il est aujourd'hui évoqué, par exemple, à la fois par des positivistes et par des jusnaturalistes, sans pouvoir pour autant parvenir à transcender le fossé séparant ces deux approches du droit.

Le constat des juristes est partagé par les sociologues et plus généralement par l'ensemble des chercheurs dans le champ du social. René Lourau, notamment, juge le concept polysémique, fuyant et problématique : il varie avec les disciplines sans jamais désigner dans une de ces disciplines un concept clairement identifiable (Lourau, 1970).

Cependant, si l'institution s'avère être un terme incontestablement polysémique, ce n'est pas pour autant un terme neutre qui pourrait appeler n'importe quel type d'analyse. Toutes les théories de l'institution présentent un certain nombre d'éléments communs, qui les caractérisent. On peut à ce stade en retenir deux : du point de vue de l'étude des phénomènes juridiques, la recherche de leur origine dans la réalité sociale; et du point de vue de la méthode de recherche, une interdisciplinarité s'appuyant sur la connexion entre les diverses sciences concernées. Ces dénominateurs communs permettent alors de faire la partition entre, d'une part, les analyses de type institutionnel, parmi lesquelles se range la théorie de l'institution d'Hauriou, et, d'autre part, des analyses d'un autre type qui peuvent se donner des institutions pour objet : par exemple la présentation des institutions publiques telle qu'elle a été recherchée il y a quelques décennies pour renouveler l'enseignement du droit constitutionnel (Duverger, 1955) ou du droit administratif (Langrod, 1966).

Revenir aux écrits de Maurice Hauriou ne doit pas laisser supposer que l'institution ou plus exactement l'analyse institutionnelle serait, comme certains se plaisent parfois à le croire, une construc- 
tion intellectuelle archaïque à laquelle on rendrait hommage à l'occasion de la présentation des pères du droit administratif, par opposition peut-être à la postérité de la notion de service public (notion qui, remarquons-le, n'est guère moins polysémique). II s'agit certes dans les deux cas d'une construction intellectuelle, d'un modèle explicatif du droit et de son rapport au social. Mais ces modèles ne sont pas figés (Espagno, 1991), notamment, pour ce qui est de l'institution, dans la formulation qu'en aurait faite Hauriou : il y a en droit un certain nombre de recherches, tout particulièrement depuis quelques années, qui lui insufflent une nouvelle dynamique.

Ces recherches démontrent l'un des caractères les plus porteurs de l'analyse institutionnelle: elles débouchent sur une réflexion transcendant les branches du droit, public ou privé, à la fois dans une perspective de théorie générale du droit, et dans un souci d'analyse du droit positif.

En revanche, elles témoignent aussi d'une faiblesse dans la diffusion de cette analyse. Si nombreux sont les juristes qui connaissent la théorie de l'institution, qui appartient à la culture juridique universitaire de base en France, le nombre de ceux qui en font véritablement un objet ou un outil de leur recherche est bien plus faible, même s'il semble progresser ; au-delà surtout de la parution d'articles spécifiques ou des recherches d'universitaires isolés, il semble bien que seules les universités toulousaines connaissent une activité ordonnée et continue en la matière: enseignements (Mazères, 1994a), recherches collectives (Marty, 1969) et surtout thèses traduisent un réel mouvement de production scientifique autour de l'institution.

On peut dater des années cinquante la naissance de ce mouvement et le situer en droit privé, avec la thèse de Michel Despax sur l'entreprise, qui poursuit les analyses de Paul Durand (Durand, 1956), ou celle de Gabriel Roujou de Boubée sur l'acte collectif (Despax, 1957 ; Roujou de Boubée, 1961). II s'étend à partir des années soixante au droit public puis à la théorie du droit, à la suite particulièrement de la thèse de Jacques Mourgeon sur la répression administrative (Mourgeon, 1967) ; il s'implante durablement à partir des années soixante-dix avec un ensemble de thèses dirigées par Jean-Arnaud Mazères (Hecquard-Théron, 1977).

Mais il faut convenir qu'en dehors de cet important pôle de travail, qui n'est d'ailleurs pas institutionnalisé puisqu'il y a un refus révélateur chez ces chercheurs d'inscrire leurs recherches dans une logique d'organisation et surtout dans une logique entre organisations, la théorie de l'institution est perçue quelque peu comme un «folklore», malgré la qualité des efforts entrepris pour la faire mieux connaître (Marty, 1969 ; Tanguy, 1991).

Lucien Sfez témoigne d'une telle perception lorsque, dans sa thèse sur Hauriou et le droit administratif, il assigne à la théorie de 
E. Millard

Hauriou et la théorie de l'institution
I'institution une place seconde et n'établit pas le lien essentiel entre cette théorie et le reste du travail d'Hauriou, semblant considérer ce dernier comme seul important du point de vue juridique.

Or, s'il y a une chose certaine avec l'institution, une chose présente chez Hauriou et chez tous ceux qui s'y réfèrent, c'est le refus d'une telle coupure.

L'analyse institutionnelle est d'abord une position épistémologique, que l'on peut présenter simplement grâce à la phrase célèbre d'Hauriou : "Un peu de sociologie éloigne du droit, beaucoup y ramène ». C'est ainsi la recherche d'une construction intellectuelle qui va au-delà de la description pour tenter de constituer un modèle explicatif s'appliquant, notamment, à l'État et au droit. Cette construction s'appuie, comme nous l'avons dit, sur deux éléments : la connexion entre toutes les modalités de la connaissance du droit et l'assignation d'une place au droit dans le social.

Car ce modèle explicatif, à la différence des théories juridiques classiques, veut tenir compte de la dimension sociale de l'État et du droit : il veut tenir compte du pouvoir. II s'agit donc de constituer une théorie du droit qui regarde le droit d'un autre point de vue que la théorie classique parce qu'elle regarde la dimension sociale du droit, mais qui soit en même temps une théorie pleinement juridique - ce que permet l'interdisciplinarité véritable -, et non un simple saupoudrage d'éléments non juridiques sur une analyse du droit - ce à quoi risque d'aboutir la « kleptomanie académique » de la pluridisciplinarité (Chevallier et Loschak, 1978, tome 1, p. 67). II est un symbole de ce choix épistémologique, dont on ne sait s'il est délibéré ou non de la part d'Hauriou: dans la salle qui porte son nom à l'Université des sciences sociales de Toulouse sont suspendus les portraits des doyens successifs de la Faculté de droit ; au milieu de cet aréopage, où chacun pose dans une posture appropriée à sa fonction et à son époque, le portrait d'Hauriou détonne: assez petit, il nous transmet l'image d'un homme peint pratiquement de dos, qui jette un regard vers la salle, au-dessus de son épaule; cet homme regarde certes la même chose que les autres juristes présents ; mais il regarde différemment.

À partir de cette dimension épistémologique, on peut retenir dans un premier temps une définition simple de l'institution dans l'analyse juridique : une forme sociale établie dans la durée.

On peut ramener le droit à une fonction par rapport à cette forme sociale, qui pourra être parfois toute l'institution, comme chez l'institutionnaliste italien Santi Romano qui assimile institution et ordre juridique (Romano, 1975), mais qui sera le plus souvent, comme chez Hauriou, un moment de l'institution.

On peut enfin citer des exemples d'organisation à appréhender comme institution : I'État, la commune, l'entreprise ou la famille.

Cependant, et on retrouve ici de manière primordiale la polysémie annoncée, le terme institution pris dans ce contexte renvoie 
nécessairement à au moins deux choses, qui ne sont pas nécessairement différentes, mais qui se complètent et déterminent l'optique qu'adopte le chercheur.

L'institution est d'une part ce qui est institué : I'organisation ; dès lors, l'institution est un concept essentiel de l'analyse, que l'on peut décrire comme objet; par certains aspects, la théorie de l'institution d'Hauriou paraît aller en ce sens. Mais d'autre part, l'institution est également le processus qui institue un groupe humain : moins que la forme instituée, elle est la constitution de cette forme dans la durée; I'accent ne porte alors pas véritablement sur l'organisation elle-même, mais sur le phénomène social de l'engendrement et de la durée de cette organisation ; le concept institution n'est ainsi plus directement l'objet de l'analyse mais son outil immédiat : tel est le sens des recherches actuelles, qui privilégient à partir de la théorie d'Hauriou l'analyse institutionnelle sur le concept d'institution lui-même.

La théorie de l'institution d'Hauriou apparaît donc, relativement à cet ensemble de recherches institutionnalistes diverses, comme un point de départ, et non pas comme un point d'achèvement. Cette situation commande l'analyse: à la présentation du concept d'institution chez Hauriou (I) doit répondre son évaluation (II).

\section{Présentation du concept d'institution chez Hauriou $^{1}$}

Le concept d'institution revêt chez Hauriou une importance capitale, tant dans les écrits de théorie générale du droit, qui lui sont directement consacrés (Hauriou 1906 ; 1925), que dans des écrits en apparence plus techniques: notes d'arrêt ou manuels (Hauriou 1910).

Pourtant, ce concept est difficile à saisir chez le doyen toulousain. D'une part, et c'est là un des traits majeurs de la pensée de cet auteur, sa place dans les écrits, son rôle dans l'explication théorique du droit, et son contenu même vont varier dans le temps en fonction de l'évolution de la réflexion d'Hauriou, capable de se « contredire» - avec honnêteté - d'un ouvrage à l'autre, voire d'une édition à l'autre. D'autre part, à aucun moment on ne trouve de présentation synthétique, exhaustive et définitive à laquelle le lecteur pourrait se reporter avec certitude. Les exposés de théorie du droit sont rédigés en termes qui peuvent nous apparaître souvent confus, avec de multiples références à d'autres sciences ou doctrines que le juriste de la fin du $x x^{e}$ siècle ne possède pas nécessairement (cela va de la théologie à la thermodynamique en passant, notamment, par la psychologie, la philosophie ou la théorie de la relativité). II faut donc pour prétendre approcher le concept tenter de mener une double approche, qui correspond d'ailleurs
Droit et Société 30/31-1995

1. Cette présentation s'appuie très largement sur les recherches de Jean-Arnaud Mazères, et notamment sur un cours professé dans le cadre du DEA de droit public à l'Université des sciences sociales de Toulouse (MAZĖrES, 1994a). 
E. Millard

Hauriou et la théorie de l'institution

2. Contrairement à ce qu'on peut d'ailleurs lire dans Le petit Robert qui est sans doute victime d'une confusion entre Maurice Hauriou et son fils André en présentant le premier comme un socialiste humaniste. parfaitement à l'épistémologie institutionnelle : une approche génétique suivra sa constitution (I.1) alors qu'une approche structurale s'attachera à en présenter l'architecture (I.2).

\section{I.1. Approche génétique}

Hauriou n'a pas eu l'inspiration ou la révélation immédiate et complète de la théorie de l'institution, pour autant qu'une théorie puisse apparaître ainsi ex nihilo. II s'est inspiré d'un ensemble de théories et réflexions qui étaient dans la discussion publique au tournant du siècle et a réagi par rapport à elles. La diversité de ces sources d'inspiration, la nécessité d'une réaction du juriste vis-àvis de celles-ci, explique en grande partie qu'il ait fallu près de vingt ans pour passer d'une première esquisse de l'institution à une théorie élaborée. On ne peut se dispenser de situer la théorie d'Hauriou par rapport à cette ambiance intellectuelle; ensuite seulement on pourra retracer l'élaboration même de cette théorie.

\section{a. Le lien avec les autres théories du social}

Sans dresser une biographie d'Hauriou (Sfez, 1966), il faut rappeler en premier lieu certains aspects de sa formation.

Maurice Hauriou est né en 1856 en Charente. II fait des études de droit à Bordeaux et se révèle particulièrement brillant dans deux matières : le droit romain et le droit civil. II n'est donc pas, de formation, un publiciste au sens moderne du terme et ce premier élément marque fortement ses analyses.

Il réussit le concours d'agrégation, unique à l'époque, et est nommé à Toulouse dans les années 1880: on lui confie alors l'enseignement d'une matière dont personne ne semblait vouloir se charger, le droit administratif. La matière était embryonnaire : on n'était pas encore à l'âge d'or du droit administratif, qu'avec son camarade d'étude et de concours, Duguit, il allait marquer. Ce deuxième élément : enseigner une matière restant à créer, a sans doute fortement compté dans l'attitude de recherche constante et de remise en cause fréquente qui a marqué l'émergence de la théorie de l'institution.

Hauriou s'inscrit dans une double filiation de pensée. Incontestablement, c'est un libéral, attaché aux principes de 1789, et méfiant vis-à-vis de tout colbertisme ${ }^{2}$. II suffit de rappeler le cri d'indignation qui marque sa note sous un arrêt célèbre quand l'État oblige les propriétaires à s'unir dans une association syndicale investie de prérogatives de puissance publique que les juges qualifient de personne publique: «On nous change notre État, on va vers le collectivisme » (Hauriou, 1900). Mais surtout, et si l'on n'a pas cela constamment à l'esprit on ne peut pas comprendre tout à fait ce qu'il présente, Hauriou est profondément catholique : il est profondément attaché à la tradition de l'Église et à la philo- 
sophie thomiste. Ceci expliquant sans doute cela, il ne parviendra jamais à se départir - et même sans doute ne s'y essaiera-t-il pas - d'un idéalisme reposant aussi bien sur la force des consciences individuelles que sur une conscience supérieure, qui n'est finalement rien d'autre que l'idée de Dieu, sous la référence d'une conscience qui est alors plus commune que collective.

Libéral, catholique, thomiste: trois éléments qui donnent un sens à sa réflexion, et qui pour une large part permettent de comprendre la recherche quasi obsessionnelle chez Hauriou d'un équilibre, entre l'État et l'initiative individuelle ou entre le mouvement et l'ordre par exemple.

Sur cette trame intellectuelle se sont greffées diverses influences.

Ce sont d'abord des influences philosophiques. Hauriou est fortement marqué par le vitalisme et par Bergson, au point d'intituler ses derniers écrits sur l'institution: Essai de vitalisme social (Hauriou, 1925), même s'il se sépare de Bergson sur divers points, notamment sur l'appréhension continue du temps.

Ce sont ensuite des influences scientifiques. Le juriste toulousain avait une curiosité aiguë de tout ce qui pouvait se faire en termes de recherches dans la sphère des sciences exactes ou expérimentales: il suivait de très près toutes les découvertes et s'intéressait aux nouvelles théories avancées, particulièrement en ce qui concerne la physique (il y trouvait notamment de quoi nourrir sa réflexion sur la notion d'équilibre). Sa curiosité ne s'arrêtait pas à de simples lectures, voire à quelques emprunts : par exemple, ayant étudié la thermodynamique, il va se passionner pour le principe d'entropie et tenter de l'appliquer au droit dans ses enseignements (Hauriou, 1899).

Tout ceci a eu certainement son importance dans la recherche qu'il mène sur l'institution. Mais cette importance est difficile à établir et quantifier davantage. II n'en va pas de même de deux autres influences, bien plus précises : la sociologie de Durkheim, et les théories juridiques sur le service public.

Avant Hauriou, Durkheim s'était attaché à la notion d'institution et à déployer une analyse institutionnelle: on sait qu'Hauriou connaissait bien la sociologie de Durkheim.

La construction de la sociologie chez Durkheim est intimement liée à une certaine conception de l'institution. Il faut partir de la définition du fait social, qui est au centre de l'épistémologie de Durkheim : un fait social se reconnaît au pouvoir de coercition externe qu'il exerce ou est susceptible d'exercer sur les individus. II y a deux éléments importants ici : ces faits sont une contrainte pour les individus car ils s'imposent à eux ; et surtout ils leur sont extérieurs. Coercition, externalité.

La question que l'on ne peut alors manquer de se poser est de savoir d'où viennent ces faits. Extérieurs à l'individu, ils ne vien- 
E. Millard

Hauriou et la théorie de l'institution nent pas de lui. Ils ne viennent pas non plus pour Durkheim de Dieu. Ils sont alors l'expression de ce que le collectif existe, et de ce qu'il a une réalité indépendante de l'individu, indépendante de sa conscience et de sa volonté. De là la formulation de l'existence d'une conscience collective, et de la nécessité d'analyser ces faits comme des choses. Et de là l'apparition de la notion d'institution, qu'on peut alors définir avec le disciple le plus proche de Durkheim : "L'institution est l'ensemble des actes et des idées tout institué que les individus trouvent devant eux et qui s'imposent plus ou moins à eux » (Mauss, 1901). Durkheim s'est référé directement à cette définition et a repris l'analyse à son compte dans la préface qu'il a donnée à la seconde édition des Règles de la méthode sociologique: «On peut appeler institution toutes les croyances et tous les modes de conduite institués par la collectivité »; et de préciser : «La sociologie est la science des institutions, de leur genèse et de leur fonctionnement » (Durkheim, 1993).

Toute l'analyse d'Hauriou va se développer à partir de cette conception sociologique, et dans un sens très clair : remettre en cause l'idée de conscience collective, point nodal de la théorie de Durkheim, en révélant la nature toute objectiviste, et qu'Hauriou n'accepte pas. Voulant redonner sa place au subjectif, il doit intégrer une idée qui semble absente chez Durkheim, pour lui faire jouer le rôle que la conscience collective ne joue plus : l'idée de puissance.

Si quelqu'un s'est inspiré de Durkheim dans une approche juridique, pour parfois d'ailleurs en faire une critique argumentée, c'est bien Duguit: la théorie du service public que l'on connaît, c'est-à-dire une théorie qui part, même si elle s'en éloigne ensuite, de la conscience collective et qui tente de rendre compte de la cohésion sociale, le démontre. Il est d'ailleurs intéressant de noter qu'à l'époque où Durkheim théorise l'institution, Hauriou, quant à lui, est sur une ligne de recherche qui est totalement celle du service public (Rivero 1956 ; Sfez, 1966). Cela montre à quel point I'opposition institution/service public, ou Hauriou/Duguit si I'on préfère, est artificielle.

L'institution, au moins dans la pensée d'Hauriou, n'est pas l'antithèse du service public. Ce sont deux moments différents de sa recherche sur le droit et le social. Ce sont surtout des réponses à des questions différentes et successives, logiquement et génétiquement : celle de la cohésion immédiate (l'identité) et celle de la cohésion dans la durée (le pouvoir). Si Hauriou critique Duguit (et partant se critique lui-même), s'il abandonne la priorité donnée aux théories du service public, ce n'est pas pour se renier, mais pour dépasser cette analyse. Ne parvenant pas à trouver la durée dans les théories du service public, il doit parvenir à l'y introduire.

II n'arrive pas en effet à trouver dans la construction duguiste une explication du temps qui passe et de la lutte contre le pouvoir 
corrupteur du temps qui passe, c'est-à-dire une explication du maintien de la cohésion du groupe dans le temps, et contre lui (Ségur, 1993). Car la cohésion ne peut pas être liée seulement à l'idée de service public, cette idée ne traduisant qu'une cohésion à un instant $t$. II faut que quelque chose maintienne la cohésion (et donc l'idée de service public qui assure la cohésion immédiate) dans le temps, dans la durée.

Il est alors clair que la théorie de l'institution d'Hauriou tente à la fois (mais c'est la même chose puisque cela se répond) l'introduction dans la sociologie de Durkheim de la puissance et l'introduction dans la théorie du service public de la durée, la puissance permettant le déploiement dans la durée.

Ces deux influences sont claires; en revanche, il manque deux pièces importantes au dossier.

D'une part, il ne semble pas que Hauriou ait eu connaissance ou ait réagi aux recherches de Santi Romano sur l'institution en Italie. Santi Romano, Iorsqu'il publie en 1918 la $1^{\text {ère }}$ édition de L'Ordre juridique, connaissait pourtant et se réfère très explicitement aux travaux antérieurs d'Hauriou (Romano, 1975). Mais Hauriou, pour sa part, ne se situe jamais ensuite par rapport à l'institutionnalisme italien, et d'ailleurs Santi Romano lui-même n'a pas intégré les recherches ultérieures d'Hauriou. II n'y a eu ici aucune confrontation entre ces deux analyses, qui représentent pourtant deux des formes les plus abouties de l'institutionnalisme juridique.

D'autre part, Hauriou se préoccupe peu du mouvement juridique normativiste et notamment des réflexions qui apparaissent en Autriche autour de Kelsen. Bien sûr, sa mort précède la parution de La théorie pure du droit (respectivement 1929 et 1934). Mais, dès 1920, Kelsen a une dimension internationale, et il est curieux (et certainement dommage) qu'Hauriou n'ait consacré que de trop rapides analyses à ce qui reste malgré tout le courant majeur en droit au XX siècle (Hauriou, 1929, préface et p. 8-12). II reste inscrit dans un débat plus ancien entre l'objectivisme, duguiste notamment, et le subjectivisme, qui marque tant le droit civil de l'époque (Gounot, 1912). II tente certes de transcender ce débat en conciliant les deux approches : en réservant une part à chacune d'elles (pour faire court, le mouvement pour le subjectif, l'ordre pour l'objectif); mais il ne parvient pas à l'appréhender dans les termes de la modernité juridique.

\section{b. Historique d'une élaboration}

On trouve dès le départ chez Hauriou une préoccupation générale concernant le social, le mouvement et le temps. Dès les écrits préinstitutionnalistes, on voit apparaître toutes les interrogations qui vont donner l'institution (Hauriou, 1896 ; 1899). Et si I'on faisait l'effort d'une analyse exégétique des manuels ou des notes des 
E. Millard

Hauriou et la théorie de l'institution années 1895-1905, on arriverait sans trop de peine à démontrer que les éléments de la théorie sont tous là, même s'ils ne sont pas saisis dans une perspective scientifique, parce qu'Hauriou n'a pas encore l'outil institution pour le faire.

Hauriou a mis en œuvre cet outil dès qu'il a renoncé à l'optique principale du service public, c'est-à-dire à partir de 1903-1904. Apparaît alors véritablement une doctrine sur l'institution ; elle va considérablement évoluer encore, sans que le projet ne soit plus cependant remis en cause.

Il faut s'arrêter sur des étapes dans cette évolution. On peut en retenir au moins trois.

La première est certainement celle de l'ébauche d'une théorie

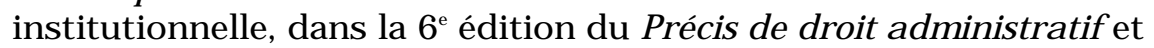
surtout dans un article de 1906, L'institution et le droit statutaire: le concept d'institution n'est pas encore clairement saisi ; mais en distinguant le droit statutaire et le droit disciplinaire, lié à la discipline organisationnelle du groupe, Hauriou se donne les premiers moyens pour une approche plus complète du droit (Hauriou, 1906).

La deuxième étape est celle de la réflexion sur une théorie institutionnelle de l'État dans les Principes de droit public (Hauriou, 1910). Hauriou y avance deux éléments fondamentaux de son approche.

- D'abord, il explique que l'on ne peut pas comprendre l'État si I'on mène une étude simplement juridique de la seule personnalité juridique de l'État, et donc de l'unité. II faut chercher avant la personne juridique de l'État, avant la personne unitaire, le pluralisme et l'équilibre entre les diverses forces sociales (forces économiques, forces politiques, etc.) qui vont aboutir à l'État. II y a donc immédiatement dans l'analyse que propose Hauriou une dimension génétique. Mais il y a aussi l'expression de la certitude qu'a Hauriou : avant l'État, donc avant la personnalisation juridique, il peut y avoir équilibre, il peut y avoir un pouvoir organisé, et donc il peut y avoir du droit. Hauriou pense particulièrement à trois éléments qui sont pour lui du droit préétatique : l'institution ; le commerce juridique, c'est-à-dire sans doute le marché au sens économique du terme; et le contrat, c'est-à-dire l'accord entre acteurs. L'État est ainsi présenté comme une construction à partir de ces éléments juridiques que sont l'institution, le marché et le contrat.

Même si Hauriou ne semble pas y faire référence, il est frappant de constater que l'on rencontre là une analyse assez proche de celle menée par Hegel sur la constitution de l'État à partir de la famille ou de la corporation (Hegel, 1940).

- Ensuite, Hauriou démontre que la puissance ne s'oppose pas au consentement. II peut effectivement y avoir à la fois des phénomènes de pouvoir et un consentement de ceux qui sont 
concernés par ce pouvoir. Cela permet à Hauriou de rechercher

autrement qu'au travers du contrat social, mythe fondateur, l'explication de l'État, aussi bien quant à sa fondation par la puissance, que quant à son acceptation par les citoyens.

La troisième étape est celle d'une réflexion fondamentale sur I'institution elle-même : La théorie de l'institution et de la fondation (Hauriou, 1925). Hauriou approfondit sa réflexion en proposant un modèle théorique qui ne concerne plus seulement l'État, mais s'applique à tout groupe organisé.

Il y a donc ici une évolution essentielle à mettre en évidence. L'administrativiste Hauriou, le juriste Hauriou s'interroge d'abord sur l'État: le service public ne lui semble pas une réponse cohérente aux questions qu'il se pose à son sujet et il met en œuvre l'approche institutionnelle à propos de l'État ; c'est l'objet des recherches de la période 1903-1910. Puis, il cherche à approfondir la notion d'institution elle-même, et a priori rien ne permet de croire qu'Hauriou le fait en renonçant à son objet: l'État; mais en s'interrogeant sur l'outil, en faisant de l'outil l'objet, il généralise une méthodologie, il fonde une épistémologie, il dégage des concepts et une analyse généralisable, qui va l'amener à «perdre » en 1925 sinon l'État, du moins la spécificité de celui-ci par rapport aux autres groupes organisés.

C'est là sans doute la force de la théorie de l'institution : sa capacité à analyser directement le groupe organisé quel qu'il soit. Mais c'est là aussi sa grande faiblesse parce que, malgré tout, l'État a une certaine spécificité dont il faut bien pouvoir rendre compte, même s'il n'est pas question pour autant de renoncer à une analyse critique et génétique de l'État. II faudra alors s'interroger sur le point de savoir si cette faiblesse est liée à l'approche de l'institution que retient Hauriou, ou si elle est liée à toute approche institutionnelle.

\section{I.2. Approche structurale}

En toute logique, il conviendrait de «radiographier » la théorie institutionnelle à chacune des grandes étapes de l'évolution intellectuelle d'Hauriou. La place nous manque ici et il semble bien que I'on puisse, en première analyse, se concentrer sur la formulation qui est donnée à la dernière des étapes que nous ayons retenues: celle de 1925. D'une part, il s'agit là d'un moment essentiel de l'analyse : celui où l'institution fait directement l'objet de l'interrogation, en elle-même, et non en tant que modèle explicatif de l'État; cette formulation paraît alors être la plus complète. Et elle est reprise, d'autre part, de manière quasi systématique par l'ensemble de ceux qui font référence à l'institution d'Hauriou, au point qu'étant connue de tous, elle semble apporter quelque clarté au milieu d'éléments plus obscurs. Malgré tout, on ne pourra pas 
E. Millard

Hauriou et la théorie de l'institution

3. Ils constituent deux types d'institutions : les institutionspersonnes ou corps et les institutions-choses. Si les premières, collectivités humaines, ne posent pas de véritable problème de conception, il n'en va pas de même des secondes. Hauriou en traite explicitement, mais sans à aucun moment définir ce qu'elles sont ; il donne cependant comme exemple le droit de propriété. II semblerait donc que les institutions-choses soient pour lui des faits institutionnels autonomes, sinon totalement indépendants du groupe humain, du moins ayant un rôle suffisamment marqué dans ce groupe ou par rapport à lui pour constituer une catégorie de l'analyse. Mais reconnaissons que le concept demeure peu opératoire.

4. Puisqu'aussi bien toute I'analyse institutionnelle d'Hauriou repose sur l'idée que ces institutions se déploient dans la durée comme une vie : elles naissent, elles se développent, elles meurent ; ainsi entend-il traiter du vitalisme social (HAURIOU, 1925). occulter les approches antérieures, ce qui nous conduira, mais c'est là déjà plus une piste quant à l'utilisation du concept qu'une présentation, à conjuguer les approches pour préciser la notion.

II n'est pas certain que l'on soit en 1925 en présence d'une véritable définition. La formulation fonctionne selon le même principe que la pseudo-définition juridique de l'État, par énumération d'éléments constitutifs. Hauriou parle d'ailleurs lui-même, en présentant cette formulation, des grandes lignes de la théorie, et non d'une définition.

Cette formulation est la suivante :

«Une institution est une idée d'œuvre ou d'entreprise qui se réalise et dure juridiquement dans un milieu social ; pour la réalisation de cette idée, un pouvoir s'organise qui lui procure des organes ; d'autre part, entre les membres du groupe social intéressé à la réalisation de l'idée, il se produit des manifestations de communion dirigées par les organes du pouvoir et réglées par des procédures. » (Hauriou, 1925, p. 96)

La formulation est pour le moins complexe et obscure. Cela n'a pas empêché bon nombre de juristes de s'y référer comme à une définition de l'institution, dont on aurait fait le tour en la citant, alors qu'il ne peut s'agir que d'une éventuel point de départ pour notre propre réflexion. II faut donc commencer par «décortiquer » cette formulation, pour y faire apparaitre les trois éléments qui, selon Hauriou, sont constitutifs des institutions ${ }^{3}$ : l'idée d'œuvre, le pouvoir de gouvernement organisé et les manifestations de communion.

On peut s'attacher dans un premier temps à chacun d'entre eux, pour comprendre les concepts qu'Hauriou place derrière ces termes; il faut surtout montrer dans un second temps comment ces trois éléments s'articulent pour réaliser la vie même de l'institution dans la durée ${ }^{4}$.

\section{a. Les éléments constitutifs}

L'institution se constitue grâce à eux. Mais il faut bien comprendre, pour ne pas commettre de contresens, qu'une même logique les unit : d'abord ces éléments sont liés car ils s'engendrent I'un l'autre; ensuite et surtout, il existe une hiérarchie entre eux. De ces deux points de vue, c'est l'idée d'œuvre qui est l'éléments majeur, contenant ou appelant les autres.

\section{L'idée d'œuvre}

II s'agit dans la pensée d'Hauriou de l'idée directrice de l'entreprise, qui devient action, qui se concrétise. Ici, Hauriou n'explique rien. II illustre simplement. II nous faut donc essayer de préciser. 
Hauriou essaie au travers de l'idée d'œuvre d'établir une jonction entre deux éléments qui sont en apparence opposés : I'idée et l'action. L'idée se concrétise par l'action afin de se réaliser dans le social. À la différence donc des analyses philosophiques classiques, de celle d'Hegel en particulier, il y a une immédiateté dans I'analyse d'Hauriou entre l'idée et sa réalisation, entre la raison et l'action. Les deux sont inséparables: pas d'idée sans action, pas d'action sans idée. De manière significative donc, on trouve ici, chez un juriste, idéaliste chrétien, le même point de départ que dans la théorie marxiste par exemple (Millard, 1987) : ce n'est sans doute pas le fruit du hasard, si l'on se réfère au projet de l'analyse sociale ; et ce n'est pas sans conséquence sur l'utilisation possible de l'analyse institutionnelle.

Ce que l'idée d'œuvre n'est pas ressort assez bien d'une brève réflexion sur les écrits d'Hauriou.

Elle n'est pas le but: le but est extérieur à l'entreprise puisqu'on cherche à l'atteindre. L'idée d'œuvre lui est, quant à elle, intérieure: il y a dans l'idée d'œuvre un élément de plan d'action, d'organisation, qui n'est pas dans le but. Elle dépasse le but pour être à la fois ce but (ce que l'on cherche à atteindre), et autre chose: les moyens pour y parvenir notamment. On peut prendre l'exemple de la protection des libertés publiques dans une démocratie pour illustrer l'analyse d'Hauriou. Protéger les libertés publiques n'est pas le but de la démocratie, mais bien l'idée d'œuvre de l'État démocratique: c'est à la fois un objectif à atteindre, et un moyen pour atteindre les objectifs de cet État. C'est dire alors que tous les moyens ne sont pas bons pour protéger les libertés, notamment parce qu'ils porteraient atteinte à ces mêmes libertés ou à d'autres droits fondamentaux. Dans l'énoncé du but (élément extérieur), il y a aussi l'énoncé des moyens (élément intérieur qui constitue l'État parce qu'il lui donne les moyens d'agir). On retrouve la même idée en droit administratif entre les prérogatives de puissance publique (moyen) permettant la recherche du but de service public: I'idée d'œuvre. L'élément important, ce n'est pas l'un ou l'autre, le service public de Duguit ou la puissance publique (Vedel, 1954), mais bien la conjonction des deux : la puissance au service du «public », c'est-à-dire au service de l'intérêt général.

Elle n'est pas non plus la fonction. La fonction correspond à ce qui est fait, à ce qui est déjà réalisé, alors que l'idée d'œuvre est tournée vers le futur: ce qui est à faire, et qui n'est peut-être même pas encore déterminé. L'idée d'œuvre dépasse à son tour la fonction en y ajoutant une part de virtuel, une appréciation temporelle, donc un élément de liberté. Dans I'analyse classique alors, la fonction, c'est l'administration du groupe, ce qui existe, alors que l'idée d'œuvre, c'est la dimension du choix politique. 
E. Millard

Hauriou et la théorie de l'institution
D'un point de vue positif enfin, l'idée d'œuvre est l'objet de l'entreprise : «C'est par l'idée et en elle que l'entreprise va s'objectiver et acquérir une individualité sociale » (Hauriou, 1925).

L'idée d'œuvre a donc pour Hauriou une dimension objective (et on retrouve ainsi une approche qui n'est pas éloignée de ce que I'on connaît chez Durkheim ou Duguit : conscience collective ou loi du milieu social). Mais, et c'est là qu'Hauriou pousse jusqu'au bout son analyse, pour que l'idée objective puisse devenir l'objet de l'entreprise collective, il faut qu'elle passe de l'objectif au subjectif, parce qu'en soi l'objectivité ne permet pas d'agir : toute action est humaine, et il faut que des individus réagissent à cette idée, qu'ils œuvrent pour elle, ou qu'ils y adhèrent. C'est justement parce que cette idée est objective qu'elle peut susciter des actions ou des adhésions qui sont elles-mêmes subjectives.

Cette relation entre l'objectif et le subjectif est fondamentale chez Hauriou. Elle doit assurément être soumise à la critique parce que, notamment, elle exclut a priori tout le rôle de l'hégémonie idéologique : I'idée n'est peut-être pas toujours aussi objective que veut le croire Hauriou, et il faudrait s'attacher à une analyse génétique de l'idéologie, ou de ce que Jacques Chevallier notamment désigne comme Code (Chevallier, 1986), pour voir comment, moins qu'objective, l'idée, c'est-à-dire finalement les valeurs dominantes qui fondent la cohésion du ou des groupes sociaux, est générée et présentée comme objective. Cette nuance importante mise à part, il n'est cependant pas inintéressant de voir comment Hauriou décrit un mécanisme essentiel pour comprendre la cohésion des groupes sociaux. Finalement là encore, malgré ses positions idéalistes qui nient l'idéologie, on retrouve un mécanisme bien saisi par Gramsci par exemple (Anderson, 1978). Et très pratiquement, le mécanisme des principes généraux du droit dans la jurisprudence administrative illustre bien l'analyse d'Hauriou. «Découverts » par le juge, ils le seraient parce qu'ils auraient une dimension objective, donc parce qu'ils préexisteraient à leur formulation jurisprudentielle, et qu'ils joueraient un rôle dans la détermination de l'idée d'œuvre de la République : droits de la défense ou principe d'égalité par exemple.

\section{Le pouvoir de gouvernement organisé}

Il découle directement de l'idée d'œuvre : il est pour sa réalisation, et à son service. Résultat d'une réaction subjective de certains individus à l'idée objective, il doit permettre à l'idée de se réaliser, et donc à l'entreprise de s'objectiver : d'acquérir une dimension sociale.

Hauriou insiste sur deux principes essentiels dans l'organisation du pouvoir de gouvernement, qu'il va reformuler dans sa logique: la séparation des pouvoirs et le régime représentatif. Si 
I'analyse classique ne retient ces deux techniques de gouvernement qu'en ce qui concerne généralement l'État et le droit constitutionnel, Hauriou, pour sa part, l'applique à toutes les institutions: l'État, bien sûr, mais également les autres institutions publiques (établissements publics ou surtout collectivités locales) et les institutions privées (entreprise, famille, etc.)

Avec la séparation des pouvoirs telle que la conçoit Hauriou, on est à l'opposé de Montesquieu. La recherche permanente de l'équilibre dans la théorie institutionnelle ne recoupe pas la distinction entre la faculté d'agir et la faculté d'empêcher, tout simplement parce que cette distinction, comme l'ensemble de l'analyse classique, est par essence structurale, atemporelle, et que ce qui intéresse avant tout Hauriou, c'est le déploiement dans la durée.

$\mathrm{Au}$ lieu donc des trois pouvoirs exécutif, législatif et judiciaire, Hauriou distingue, avec son vocabulaire qui a fait couler beaucoup d'encre : la compétence intuitive de la décision exécutoire, la compétence discursive du pouvoir délibérant et le pouvoir de suffrage ou pouvoir d'assentiment.

Brièvement, de quoi s'agit-il ?

La compétence intuitive de la décision exécutoire correspond au pouvoir d'une minorité, qui a l'intuition de l'idée, et qui va agir normativement, par une décision qui aura vocation à être exécutée (exécutoire). On est encore ici dans une logique de l'intuition et de l'irrationnel qui va permettre le passage de la découverte minoritaire à la réalisation majoritaire : ce n'est alors pas une logique du contrat d'association, mais bien plutôt une logique de l'acte de fondation, comme le rappelle le titre de l'article de 1925 : La théorie de l'institution et de la fondation.

La compétence discursive traduit le fait que l'idée, telle qu'elle est perçue par le pouvoir exécutif (et donc éventuellement traduite en norme), va être soumise à un débat. On passe alors de l'intuition exécutoire à la rationalité du débat ${ }^{5}$. Mais on est encore dans la sphère minoritaire: ce n'est pas le groupe qui discute, mais une minorité de sujets qui ont conscience de l'idée.

II y a incontestablement ici chez Hauriou un certain réalisme qui réfute le mythe du contrat social pour tenter de saisir comment se constitue pratiquement la vérité de l'institution, dans des sphères finalement très limitées du pouvoir.

Le suffrage ou l'assentiment fait passer l'idée de la sphère minoritaire au groupe majoritaire. Hauriou a la conviction que le collectif, la masse, n'a pas de possibilité d'action, mais seulement une possibilité de réaction : elle ne peut pas réellement débattre sur une idée, ni même la formuler collectivement ; elle peut seulement réagir positivement ou négativement, l'approuver ou la désapprouver une fois celle-ci débattue ou mise en forme.

Ceux qui se sont un tant soit peu interrogés sur la démocratie, l'élection, le référendum et le fonctionnement des régimes politi-
Droit et Société 30/31-1995

5. Cette analyse doit être rapprochée de celle de l'éthique de la discussion par HABERMAS (1987). 
E. Millard

Hauriou et la théorie de l'institution ques, même (et peut-être surtout) démocratiques, devraient convenir que, même si cela nous gêne légitimement parce que cela bouscule quelque peu certaines idées reçues, cette analyse du rôle du peuple n'est pas totalement fantaisiste. II est clair par exemple qu'à I'heure actuelle l'initiative des textes provient presque toujours de l'exécutif, que la discussion est partagée entre un collège exécutif (gouvernement) et celui des assemblées législatives, et que c'est seulement l'approbation ou la désapprobation qui est réservée, le plus souvent sans réelle discussion, au législatif (que l'on pense au mécanisme de l'article 49-3 de la Constitution ou à celui du vote bloqué) ou au peuple (de manière directe : référendum, ou indirectement a posteriori : élection).

Grâce à cette conception temporelle de la séparation des pouvoirs, Hauriou peut se dépêtrer du traditionnel piège lié à la notion de représentation.

Le régime représentatif est une organisation du pouvoir qui permet aux organes de l'institution d'exprimer la volonté du corps qu'elle constitue. Un corps n'est rien sans ses organes qui, seuls, lui permettent d'agir parce qu'ils lui fournissent une volonté qui sera celle du corps (Carré de Malberg, 1962).

Le corps n'existe, n'agit, ne veut que par ses organes. Encore faut-il qu'ils veuillent pour lui, et non pour eux. C'est ce que permet le régime représentatif. Et logiquement alors, dans la théorie présentée par Hauriou, la représentation n'est pas un rapport entre personnes : représentantes et représentées, mais entre des personnes et une idée représentée : celle qui fonde le corps.

Dans cette optique, il y a représentation lorsque le gouvernement et les membres du groupe ont la même idée d'œuvre, c'est-àdire se font la même idée de ce qui les unit et de ce qui doit les unir.

Voilà qui tempère, et de manière importante, le réalisme quelque peu césariste de la séparation des pouvoirs. En effet, les gouvernants sont ici dans un état de totale précarité. À tout moment peut apparaître un décalage entre l'assentiment des gouvernés à l'idée et l'intuition que s'en font les gouvernants, qui peut introduire une distorsion au sein de la représentation. In fine, on retrouve là encore une parenté complète avec les interrogations de Duguit qui développe, sans passer par l'institution, la même approche au travers de la règle sociale. La loi positive chez Duguit, la décision exécutoire chez Hauriou, c'est-à-dire la règle posée qui doit être exécutée, n'ont aucune autorité par elles-mêmes : elles n'acquièrent cette autorité qu'à partir du moment où elles sont conformes à la règle de droit générée par le milieu social chez Duguit, à l'idée d'œuvre chez Hauriou. Même si chez ce dernier, le privilège du préalable - pièce essentielle associant la puissance et la durée - conditionne cette possible contestation, ou du moins en aménage l'exercice, une conformité entre le pouvoir politique et les 
valeurs du groupe est nécessaire dans les deux cas : une communion.

\section{Les manifestations de communion}

La réalisation de l'institution nécessite ainsi des manifestations de communion des membres du groupe, aussi bien que des organes de gouvernement, soit en l'idée d'œuvre à réaliser, soit en celle des moyens à employer. L'idée directrice passe à nouveau ici à l'état subjectif, par l'adhésion de chacun des membres du groupe à l'idée, qui l'assimile et la fait sienne. Cette communion traduit l'immédiateté entre l'objectif et le subjectif, le collectif et l'individuel, le groupe et chacun de ses membres.

Hauriou propose deux illustrations de ces manifestations.

- Il en voit d'abord un exemple dans les grands mouvements populaires qui accompagnent la fondation d'institutions politiques et sociales nouvelles: au moment de leur émergence. L'adhésion est ici immédiate, les trois pouvoirs sont concomitants. Hauriou pouvait penser ici notamment à la Révolution française (Fête de la Fédération par exemple), ou à la fondation de certains mouvements politiques. On pourrait aussi y voir la théorisation d'une certaine forme d'acquisition de légitimité, comme celle de de Gaulle à la Libération, en 1958 voire le 30 mai 1968. Mais on ne peut s'empêcher d'y voir également une prémonition du cérémonial sinistre dont les régimes fasciste ou hitlérien seront friands: Marche sur Rome, Parades de Nuremberg, etc. II y a dans cette conception une grande part de symbolisme: Sartre, dans sa propre vision de l'institution, s'attarde lui aussi sur de telles manifestations qui témoignent de ce qu'il appelle le groupe en fusion, quand chacun est le groupe et que le groupe est chacun (Sartre, 1960).

- Malgré un symbolisme évident, ces manifestations demeurent exceptionnelles. Plus fréquemment, l'institution repose sur une communion froide liée à son fonctionnement: dans l'acceptation par les divers membres du groupe non pas de telle ou telle décision ou action déterminée, mais du fonctionnement général de l'institution, c'est-à-dire dans l'acceptation de la règle du jeu. Hauriou voit cette communion par exemple dans le fait que la minorité accepte la décision de la majorité, non pour elle-même, mais parce qu'il s'agit de l'exercice du pouvoir au service de l'idée. II s'agit donc là d'une communion qui se réalise à tout instant et qui se reproduit à tout instant : une communion au pouvoir, qui assure la durée.

À l'évidence, c'est ici que l'idéalisme d'Hauriou est le plus fort, et que sa théorie est la plus faible : il évacue totalement la dialectique entre l'instituant et l'institué, entre le peuple et le pouvoir, dans une sorte de syncrétisme des gouvernants et des gouvernés 
E. Millard

Hauriou et la théorie de l'institution qui n'est guère plus satisfaisant finalement que la vieille idée de contrat social. Mais c'est là sa logique: une logique de l'équilibre permanent quoique évolutif, dans le processus d'institutionnalisation, dans la durée.

\section{b. L'articulation des éléments : la durée}

Les trois éléments de l'institution s'articulent selon un mouvement essentiel qui permet la durée dans le milieu social : Hauriou le désigne comme l'intériorisation.

Ce mouvement, qui va de l'objectif au subjectif, met en œuvre deux phénomènes successifs qui caractérisent la dynamique des institutions: I'incorporation et la personnification. L'idée est incorporée par des individus du groupe qui, au travers des actes de gouvernement ou des procédures, par exemple, agissent pour l'idée. C'est le gouvernement minoritaire et intuitif. Il est intériorisé lorsque des manifestations de communion apparaissent par lesquelles les membres du groupe font leur l'idée directrice, quand elle ne leur est plus extérieure, mais que l'idée objective s'est réfractée dans les consciences individuelles, pour reprendre les termes d'Hauriou. Ce dernier mouvement conduit à faire naître une institution conçue comme un véritable corps. Le gouvernement ne cesse pas, mais à l'intuition minoritaire succède l'assentiment majoritaire.

C'est dans cette articulation des éléments que l'on peut saisir le droit dans l'optique institutionnelle. Dans la théorie juridique classique, c'est le droit, c'est-à-dire un acte juridique, qui crée les institutions : acte juridique de la fondation, contrat d'association, Ioi constitutionnelle, etc. Simplement, il reste une question en suspens : qu'est-ce qui, à l'origine, crée la première institution de toutes, celle qui contient toutes les autres puisqu'elle contient les actes juridiques qui peuvent les créer, l'État? La théorie juridique classique établit ici une césure entre, d'une part, son explication de la création juridique des institutions non étatiques et, d'autre part, son absence d'explication juridique pour la création de l'État ; cela devient une question politique ou métaphysique, qui ne concerne pas directement le juriste, du moins qui n'est pas une question juridique: le juriste raisonne dans le cercle clos de l'État, sans s'interroger sur sa juridicité même. Cela veut donc dire qu'il ne s'interroge pas véritablement non plus sur la juridicité des actes juridiques reconnus par l'État, ceux notamment qui créent l'institution non étatique, puisque ceux-ci tirent leur caractère juridique de la juridicité étatique.

En articulant les éléments de l'institution, Hauriou déploie une analyse toute différente. Pour lui, et c'est sans doute là l'élément fondamental de toute approche de type institutionnel, le droit ne peut strictement rien créer. II n'est pas une force d'initiative ou d'action, il n'est qu'un élément de réaction. La création ne dépend 
pas du droit, mais de l'action humaine. Cependant, cette action, individuelle ou collective, se heurte à un obstacle irrémédiable: l'action contraire du temps corrupteur. L'impulsion se dissout dans la durée, jusqu'à disparaître.

Hauriou utilise l'image classique du jet d'eau, qu'il emprunte au vitalisme de Bergson. L'initiative humaine, le pouvoir intuitif sont comparés à la force qui fait jaillir l'eau vers le haut. Cette force n'est pas juridique : elle est immédiatement humaine et sociale. Mais pour que l'eau ne retombe pas, il faut qu'une autre force, permanente, réagisse à la tendance naturelle de l'eau à retomber. Il en va de même dans l'institution. Pour que le groupe ne se dissolve pas, pour que l'idée ne se corrompe pas avec le temps, il faut qu'une force réagisse à l'effet corrupteur du temps : c'est là le rôle du droit. Ainsi ce n'est pas le droit qui crée l'institution, mais l'institution, et chaque institution, qui génère nécessairement du droit par le processus qui crée l'institué contre la tendance de l'instituant à se dissoudre dans la durée.

Cette approche se retrouve dans la classification essentielle qu'Hauriou établit au sein des règles juridiques, entre le droit statutaire, qui garantit les droits individuels des membres du groupe dans l'institution, et le droit disciplinaire, qui garantit la cohésion du groupe en mettant en place une discipline organisationnelle: droits fondamentaux, police collective (Hauriou, 1906).

Et c'est dans cette approche que l'on peut saisir l'irréductibilité des doctrines de l'institution et du service public. Chez Duguit, le service public est censé poursuivre la cohésion sociale, mais sans réel moyen juridique d'action : l'État est une collection de services publics. Alors que chez Hauriou, cette cohésion est recherchée en mettant des moyens juridiques de police au service de la cohésion : la puissance publique au service de la cohésion collective. Chez Duguit, il y a une pétition de cohésion semble-t-il, qui ne tient pas compte de la réalité sociale et du temps, alors que chez Hauriou, cette dimension est prise en compte au travers de la puissance, doublement limitée cependant: par les droits statutaires des membres, et par l'idée d'œuvre, constituant ou fondant l'institution.

À l'inverse des représentants de la théorie subjectiviste classique, Hauriou pose finalement la question du groupe à l'envers. Là où le subjectivisme essaie d'appliquer au groupe la problématique de l'individu au travers de la personne morale, en constituant le collectif juridique à partir de l'individu et à son image, Hauriou part, quant à lui, directement du groupe. Pour lui, le collectif est directement dans l'individu : il n'y a pas de césure entre le groupe, qui nous serait extérieur, et nous. L'idée objective n'est pas perçue ici, à l'image de la conscience collective de Durkheim ou de la règle émanant du milieu social de Duguit, comme la moyenne des consciences individuelles, ou comme l'idée se dégageant majoritaire- 
E. Millard

Hauriou et la théorie de l'institution ment des consciences individuelles, c'est-à-dire comme l'objectivation de consciences subjectives. Immédiatement objective, elle est directement commune aux individus qui la perçoivent et la font leur.

C'est là d'abord la déclinaison d'une idée philosophique plus générale, qu'on retrouve aussi bien dans une certaine pensée chrétienne que dans les écrits du jeune Marx, selon laquelle il ne faut pas voir en I'homme un animal simplement politique, mais avant tout un animal social. Mais l'affirmation va plus loin puisqu'elle conduit Hauriou à mener une analyse très touffue et là encore quelque peu confuse, de type psychologique, dans laquelle il compare la psychologie sociale et la psychologie humaine, et de laquelle il ressort que pour Hauriou, en dernière analyse, l'être humain lui-même peut-être abordé comme une institution. La dimension sociale est donc considérée par Hauriou comme étant avant tout une dimension psychologique. L'affirmation est a priori surprenante. Mais si la comparaison entre l'homme et le groupe institutionnalisé tel qu'Hauriou nous l'a décrit paraît rapide, et s'il est assez difficile de retrouver dans I'homme les éléments exacts qui constituent l'institution, il faut malgré tout noter que, de manière sans doute significative, alors que la majorité des juristes oubliaient très rapidement la théorie de l'institution dans les années trente, c'est avec les psychiatres d'une part, la pratique pédagogique d'autre part, que l'institution est réapparue, dans une problématique fortement empreinte d'une analyse psychologique et sociale. Pour être confuse, l'intuition du doyen toulousain n'était peut-être pas aussi saugrenue qu'elle peut paraître à certains. Et il semble bien que, si on la décrypte, on ne soit pas si loin de certaines propositions avancées par des psychanalystes, et particulièrement par Lacan, sur l'appréhension du social dans la constitution de la personnalité (Lacan, 1980). Ce décryptage appelle donc directement une évaluation critique de la théorie d'Hauriou.

\section{II. Évaluation critique de la théorie d'Hauriou}

Les travaux d'Hauriou ont finalement inspiré des recherches dissemblables et son analyse a été orientée dans des sens parfois incompatibles entre eux ; cela a pu, tout autant que la complexité des écrits d'Hauriou, rebuter plus d'un lecteur. Pourtant, sous réserve d'être rénovée, et sous réserve de savoir ce que l'on veut en faire, la théorie institutionnelle semble susceptible d'éclairer bien des points sur lesquels achoppe le juriste. Après avoir dressé un rapide inventaire de I'héritage (II.1), nous essaierons de proposer quelques pistes pouvant orienter une utilisation actuelle de cette analyse (II.2). 


\section{II.1. Un héritage partagé}

La grande majorité des juristes semble avoir renoncé depuis longtemps à une recherche systématique sur l'institution : sans juger de cette attitude, le constat ne paraît guère être contestable. On peut cependant isoler, dans les travaux minoritaires, trois ou quatre grands courants se réclamant, à un titre ou à un autre, en tout ou en partie, de l'institutionnalisme : un courant qui peut être qualifié d'idéaliste a fait évoluer la théorie de l'institution vers la métaphysique ; à ce courant peuvent être rattachés d'autres auteurs, dont Carl Schmitt est l'archétype, qui se sont servis de la théorie institutionnelle pour construire une théorie de l'action politique; un deuxième courant se réclame du positivisme et, dans une curieuse tentative de syncrétisme, veut concilier une approche institutionnelle et le normativisme; enfin certains juristes ont repris l'analyse institutionnelle à la fois à partir des travaux d'Hauriou et à partir d'autres recherches institutionnelles menées en dehors du droit dans une perspective matérialiste.

Si ces courants se réclament tous de l'institutionnalisme, ils se rattachent aux recherches d'Hauriou de manières très variables. Deux d'entre eux paraissent assurer la postérité de l'analyse, soit en développant sa logique idéaliste, soit au contraire en la propulsant dans une logique matérialiste. À l'inverse, le positivisme institutionnaliste et l'institutionnalisme schmittien semblent constituer des recherches relevant d'autres problématiques.

En effet, le positivisme institutionnaliste, dont les écrits de MacCormick et Weinberger fournissent l'illustration la plus connue, s'inscrit radicalement dans une approche normativiste (MacCormick et Weinberger, 1992). Les auteurs se réfèrent davantage à la philosophie analytique anglo-saxonne, et notamment à la théorie des faits institutionnels proposée par Searle à la suite d'Anscombe (Searle, 1972), qu'ils reformulent d'un point de vue critique (MacCormick et Weinberger, 1992, p. 115-121), qu'à la théorie institutionnelle d'Hauriou.

Même si les auteurs reconnaissent à l'égard du juriste français « une dette partielle» (MacCormick et Weinberger, 1992, p. 31), c'est pour considérer malgré tout que son approche «repose, de façon contestable, sur une approche vitaliste et idéaliste qui affirme la priorité du pouvoir et de l'idéologie sur des fonctions sociales » (Id.).

S'il ne fait aucun doute que l'analyse d'Hauriou est fortement marquée par l'idéalisme et le vitalisme, le lien qu'établissent MacCormick et Weinberger entre ce caractère et la prise en compte du pouvoir doit être souligné : en excluant une fois de plus le phénomène du pouvoir du champ de l'analyse juridique, les auteurs démontrent que leur recherche est bien, comme ils l'affirment d'ailleurs, une «nouvelle approche du positivisme juridique » (Id.) 
E. Millard

Hauriou et la théorie de l'institution

6. Voir de ce point de vue la pré face de Jean-Arnaud MAZĖRES à notre thèse dans sa version publiée, ainsi que l'avant-propos de Marie-Anne COHENDET (MILLARD, 1994). et non une analyse de type institutionnel telle que nous l'avons jusqu'ici abordée. L'institution est pour eux une référence au sein de l'analyse normative, et non une modalité permettant l'élargissement de cette analyse en obligeant à la repenser. En ce sens, l'apport de ces auteurs peut apparaître restreint si l'on considère certaines recherches positivistes qui essaient de se situer sans détour par rapport à l'analyse institutionnelle (Bobbio, 1971).

On ne peut dire à l'inverse de la pensée de Schmitt qu'elle ignore le pouvoir. Cette pensée est maintenant bien connue et Olivier Beaud a longuement mis en évidence la manière dont Schmitt s'était inspiré d'Hauriou (Beaud, 1993).

On comprend aisément ce qui a pu séduire le juriste allemand dans la théorie institutionnelle : une théorie de la puissance, de la durée, qui ramène le consentement populaire à des manifestations de communion avec le pouvoir, et qui fait de l'homme providentiel l'acteur politique légitime.

La lecture de Schmitt n'est pas unique : on dit notamment que Salazar avait fait d'Hauriou un de ses auteurs fétiches; et on retrouve chez des auteurs dont l'engagement démocratique est indéniable une même fascination pour cette forme d'institutionnalisme (Capitant, 1982).

D'un point de vue simplement scientifique, il semble pourtant que cette lecture des écrits d'Hauriou soit extrêmement réductrice, en ramenant finalement ce qui doit constituer une analyse du social à une « recette » de l'action politique ${ }^{6}$. II est pourtant indéniable que le doyen Hauriou n'a pas tout fait pour prévenir une telle lecture, qui n'est peut-être rien d'autre que le fruit d'un idéalisme caricaturé.

\section{a. L'idéalisme institutionnel}

Hauriou évoluait dans un milieu très idéaliste, fortement marqué par le christianisme. L'institution a fait son lit dans ce milieu, mais dans un sens de plus en plus doctrinaire, qui détache la démarche d'une recherche scientifique analysant le social pour lui conférer une dimension métaphysique.

Cette évolution est d'abord le fait d'Hauriou lui-même. II n'est pas excessif de considérer qu'Hauriou a pu être pris de vertige en observant son œuvre, pour deux raisons au moins.

D'une part, d'un point de vue politique, Hauriou, catholique libéral, s'accommodait sans doute assez mal des possibilités protestataires ouvertes au peuple par la procédure d'approbation/désapprobation qu'implique sa conception du régime représentatif : parti à la recherche de l'équilibre, de l'ordre et de la durée, le voilà proposant une théorie susceptible de déboucher sur la contestation permanente. D'autre part et surtout, en analysant immédiatement l'institution (Hauriou, 1925), il a perdu ce qui était à ses yeux essentiel : I'État. Si en effet le mécanisme de l'institution s'applique 
à tout groupe, la présentation achoppe sur une question que la théorie de l'institution ne peut éviter de se poser : où est alors l'État transcendant, celui qui est au-dessus du champ social, et qui le régit entièrement ? Comment, autrement dit, arriver à concevoir le modèle susceptible de fédérer les différentes institutions : entreprises, familles, syndicats, associations, collectivités diverses, dans une institution qui les englobe.

À ce stade, le mécanisme institutionnel ne fonctionne plus. Ou bien on débouche sur une assez improbable conception anarchiste du droit et du social, sur laquelle butent notamment les théories de l'autogestion: ce n'est pas là le sens des recherches d'Hauriou. Ou bien il faut introduire d'autres éléments, hétérodoxes par rapport à l'idéalisme, comme notamment la dialectique entre l'instituant et l'institué en lieu et place de l'équilibre et de l'assentiment, qui entraîneraient assurément Hauriou du côté du marxisme notamment : c'est alors la raison même de sa recherche, l'équilibre, qui s'effacerait. En toute hypothèse, en cherchant la cohésion sociale, Hauriou découvre la fragmentation sociale. Et cela, il ne peut ou ne veut l'admettre.

La seule solution pour sauver l'essentiel de la construction théorique est alors d'ordre métaphysique et transcendant: Hauriou abandonne ses prémisses scientifiques pour changer de logique, en réintroduisant l'État au-dessus du champ social, et en le faisant échapper sinon à la logique institutionnelle dans le cadre d'une analyse interne, du moins à la logique des institutions au sein d'une analyse sociale globale. Coexistent alors deux mouvements, sans lien réel entre eux: l'État comme institution d'une part, et d'autre part les autres groupes pris également chacun en ce qui le concerne comme institution. Mais aucun lien de type institutionnel n'est établi ni même recherché entre l'État et les groupes. La logique institutionnelle ne saisit plus que les individus composant chacune de ces institutions, et non pas les relations entre leurs collectifs.

Cela est très net notamment dans la seconde édition du Précis de droit constitutionnel (Hauriou, 1929), dont les 200 premières pages, consacrées aux «facteurs constitutionnels», contiennent une théorie de l'État très en retrait sur les analyse de 1925, parce qu'elle est articulée principalement autour de la puissance.

D'un point de vue pratique, cela a contribué à la diffusion d'une image quelque peu limitée de la théorie institutionnelle: comme il s'agit là des dernières pages d'Hauriou, et qu'elles sont accessibles, nombreux sont les juristes qui y ont vu l'essentiel de ce qu'a écrit le doyen toulousain, et qui n'ont retenu de son œuvre que la puissance ; et cela tend à faire accroire qu'il y aurait d'un côté un philosophe politico-juridique un peu «farfelu », l'institutionnaliste Hauriou, et de l'autre l'administrativiste rigoureux et génial qui a créé avec Duguit le droit administratif moderne et an- 
E. Millard

Hauriou et la théorie de l'institution noté les arrêts classiques du Conseil d'État. Or les deux ne sont pas séparables. S'il existe une séparation au sein de la recherche rapportée, ce n'est pas entre l'institution et le droit administratif, mais, peut-être, entre, d'une part, le juriste qui cherche jusqu'en 1925, et qui cherche aussi bien au travers de la théorie de l'institution qu'au travers des analyses techniques (ce n'est pas un hasard si Hauriou récuse, par exemple, le concept d'unilatéralité à propos des actes administratifs, et utilise celui de décision exécutoire, qui provient - ou qui implique - l'analyse institutionnelle de la normativité) ; et d'autre part celui qui, sur la fin, réticent à accepter les conséquences de sa logique de recherche, tente de refermer la boite de Pandore en reconstruisant le classicisme de l'analyse juridique sur les cendres de l'institution.

Car, d'un point de vue théorique, ce retour à l'État est bien un renoncement : il n'entraîne pas un retour à l'analyse institutionnelle de l'État déployée dans les Principes de droit public (Hauriou, 1910), qui permettrait peut-être de conjuguer institution et État, mais bien une juxtaposition de l'analyse institutionnelle et d'une analyse très classique, qui conduit Hauriou à discerner l'institution primaire (l'État) d'institutions secondaires, sans chercher à établir autrement que par une transcendance quasi axiomatique le lien entre les deux.

II convient malgré tout de reconnaître que si ce revirement est très net après 1925, il n'était pas imprévisible. Toute l'analyse des éléments constitutifs de l'institution a montré par exemple combien l'approche d'Hauriou n'arrivait pas à se départir d'une volonté a priori de trouver un équilibre, et la communion entre les membres du groupe.

Cet idéalisme sera accentué par les disciples d'Hauriou et nous pensons qu'il explique, en grande partie, le désintérêt progressif des juristes pour la théorie de l'institution. On pourrait multiplier les exemples de cette évolution : il suffit de mentionner le cas le plus connu, celui de Georges Renard, qui élabore, aux côtés d'articles théoriques remarquables, sur la notion de droit constitutionnel notamment (Renard, 1933), un essai d'ontologie juridique fondé sur l'institution qui laisse rêveur par sa description/ prescription d'une société «anarcho-christiano-corporative » toute faite d'institutions qui s'emboîtent les unes dans les autres (Renard, 1930).

Même si les écrits de cet auteur inspirent encore des travaux originaux sur l'institution (Dubouchet, 1993), ils sont finalement révélateurs des difficultés qu'ont les disciples d'Hauriou, à sa suite et pour autant qu'ils en aient la volonté, ce dont on peut douter, à se défaire de l'approche idéaliste pour saisir la dialectique sociale. C'est ce qu'a tenté de faire le courant matérialiste ou, si l'on préfère, réaliste. 
II n'est pas innocent que ce soit d'abord en dehors de la sphère des juristes que l'institution fasse l'objet d'une réflexion innovante, sans d'ailleurs le plus souvent que les acteurs fassent référence à Hauriou qu'ils semblent peu connaître (Hess et Savoye, 1993) - ce qui, entre parenthèses, donne à réfléchir sur la nature objective des idées. Ce n'est qu'ultérieurement que des juristes feront à leur tour un pareil effort.

Très schématiquement, l'institution est saisie d'abord comme outil dans le cadre d'une réflexion sur une pratique psychiatrique (Tosquelles, 1969) puis psychanalytique (Deleuze et Guattari, 1972) et enfin pédagogique (Oury et Vasquez, 1967) ; chaque fois, une réflexion est menée sur un groupe, dans un milieu relativement clos, qui génère un fonctionnement relativement autonome.

Au delà, cette approche est élargie par la réflexion philosophique et sociologique (Deleuze, 1955 ; Sartre, 1960 ; Lourau, 1970).

Deux éléments caractérisent fortement ces analyses. D'une part, l'accent est mis sur une volonté d'analyse scientifique du social, et non sur la construction théorique d'un devoir-être : quand cette condition épistémologique est oubliée, l'échec est irrémédiable, comme chez Sartre qui n'arrivera jamais à faire paraître (parce qu'il n'arrivera jamais à concevoir) le tome 2 de sa recherche sur la dialectique des groupes (Sartre, 1960). D'autre part et surtout, on n'occulte plus, et au contraire on centre l'analyse sur ce point, le rapport dialectique entre l'instituant et l'institué. L'institution est ainsi davantage comprise comme le processus instituant que comme l'objet institué. À l'inverse des approches idéalistes, l'équilibre n'est pas présumé: les contradictions du social sont traquées parce que ce sont elles qui révèlent le sens du processus. La recherche essaie ainsi de comprendre comment l'institué, quel qu'il soit, peut échapper à la tendance bureaucratique qui guette toute institution afin de ne pas exclure l'instituant, c'est-à-dire comment l'individu peut ne pas être extérieur au gouvernement du groupe auquel il appartient: ainsi apparaît l'idée fondamentale d'une évolution dialectique dans la durée, d'une auto-institution permanente du groupe.

C'est à partir de ces refondations de l'analyse institutionnelle que la démarche va être reprise par des juristes, comme par exemple Jean-Arnaud Mazères ou Jacques Chevallier. Cette relecture de l'institution nous semble désormais pouvoir constituer, pour les juristes, un champ d'investigation particulièrement prometteur.

\section{II.2. Pistes pour une utilisation actuelle}

Jacques Chevallier a parfaitement présenté dans quel état d'esprit le juriste doit aborder l'analyse institutionnelle (Chevallier, 1981). S'appuyant sur Feyerabend, il plaide pour un opportunisme 
E. Millard

Hauriou et la théorie de l'institution

sans scrupules, qui ne retient quant à cette analyse que les critères de l'opérationalité et de l'efficacité : il ne s'agit pas d'en faire une doctrine ou une théorie, mais un outil. Bien moins que la théorie d'un concept polysémique: I'institution, l'analyse institutionnelle est avant toute chose une volonté épistémologique.

Cet outil est utilisable avec profit, si on a conscience de ses limites. L'analyse institutionnelle n'explique pas tout, et il y a bien des éléments qui intéressent le juriste qu'elle n'explique pas : le processus décisionnel par exemple (Chevallier, 1981). Mais il y a aussi des faits sociaux nombreux que l'analyse institutionnelle est seule capable d'expliquer : tel est notamment le cas des processus d'institutionnalisation, c'est-à-dire des processus qui aboutissent à un phénomène institué (comme le droit) parce qu'elle est la seule à combiner le point de vue du tout (par exemple : le collectif) et des parties (par exemple: l'individu) en transcendant les frontières rigides entre les matières ou les points d'observation.

L'analyse institutionnelle essaie dès lors de suivre et de comprendre ces processus, c'est-à-dire d'analyser ce qui est institué (comme un ordre juridique) à partir de l'instituant : saisir le social et la dialectique instituante dans le processus de l'institution. II y a donc ici une différence à la fois avec l'analyse d'Hauriou, à laquelle on a reproché, de manière peut-être excessive, mais à partir de fondements réels, de considérer le droit comme un simple épiphénomène de l'institution (MacCormick et Weinberger, 1992), et avec celle de Santi Romano qui assimile droit, institution et ordre juridique (Romano, 1975).

Le juriste peut alors considérer l'institué comme renvoyant à trois éléments. En fonction de son champ d'étude (droit public, droit privé, etc.), il pourra favoriser dans l'analyse l'un de ces éléments, sans pour autant pouvoir exclure les autres, car cela ne pourrait se faire qu'en renonçant à l'analyse institutionnelle :

- l'État pris immédiatement comme institution: c'est là l'analyse institutionnelle classique en droit public, et c'est, comme nous l'avons vu, celle que voulait privilégier Hauriou puisqu'il est parti de cette analyse et, l'ayant perdue, y est revenu. Mais plus généralement, c'est aussi une analyse plus théorique et critique de l'ordre juridique étatique dans le processus institutionnel de l'État ou par rapport à lui qui est alors envisageable ;

- telle institution déterminée. Elle peut relever du droit public: les collectivités locales par exemple, d'un point de vue général (Mazères, 1990 ; Doat, 1994) ou sur des questions spécifiques (Ortiz, 1992) ; elle peut relever du droit privé, comme l'entreprise (Durand, 1956 ; Despax, 1957) ; surtout est mise en évidence une logique transcendant les discriminations «public/ privé » et «État/institutions non étatiques » pour saisir directement des phénomènes institutionnels comme ceux 
de la personnalisation (Linditch, 1991), de la réglementation (Hecquard-Théron, 1977) et de la répression (Mourgeon, 1967), ou pour situer juridiquement des concepts que ces discriminations occultent, comme ceux de marché et de Nation (Mazères, 1994b) ;

- les relations instituées ou non entre institutions, car c'est là que réside le maillon faible de l'analyse institutionnelle, et car c'est là que l'on passe de l'analyse statique d'une institution à la dynamique du processus d'institutionnalisation. Ici apparaissent la dialectique des groupes et la prise en compte du fait que chaque individu n'est pas saisi par une seule institution, mais par plusieurs (famille, État, entreprise, syndicat, etc.), ce qui peut susciter des contradictions. Ce sont ces contradictions que tend à gommer l'institué à ce stade, en reconstruisant le statut juridique de ces relations et de ces institutions: relevantes ou irrelevantes (Romano, 1975 ; Millard, 1994). Ce sont donc ces statuts et l'ensemble des groupes institués ou s'instituant que l'on vient d'évoquer qu'il faut essayer de mettre en relation, dans une perspective critique: par exemple l'État et la famille (Millard, 1994).

Cela implique que l'on déploie une logique de connexion, entre différentes disciplines et entre différents points d'observation. En partant alors de l'analyse du droit tel que nous l'avons appréhendé à la suite d'Hauriou, c'est-à-dire d'un élément de réaction dans le temps lié au processus d'institution - et non pas donc un élément de création des institutions -, on va pouvoir, par exemple, conjuguer dans un même mouvement trois niveaux d'analyse que nous ne détaillons qu'aux seules fins de nous faire mieux comprendre :

- le niveau social, ante juridique dans une perspective génétique, extra juridique dans une perspective qui analyse la part interne du droit. On s'attachera alors à la naissance des institutions ; mais surtout, pour comprendre la durée, on analysera la fonction intégratrice du droit, qui permet à l'institution de fixer en son sein le statut qu'il donne aux institutions qui lui sont extérieures (dimension dialectique entre institutions). Pour ce qui est alors notamment de l'État, il faudra rechercher pourquoi et comment l'institution État arrive à se présenter comme l'institution globale, qui est supérieure à toutes les autres, et qui les englobe. Et au-delà de l'État, une réflexion, qu'on trouve d'ailleurs par exemple chez Georges Scelle, peut être menée concernant le droit international (Scelle, 1948). L'idée est donc celle d'une confrontation entre ce qui ressort du discours normatif étatique (à propos de la souveraineté par exemple) et ce que révèle la généalogie institutionnelle de l'Etat pour poser la question trop souvent éludée du caractère juridique du droit et de l'État ; 
E. Millard

Hauriou et la théorie de l'institution
- le niveau strictement interne de ce discours normatif. Une analyse positiviste, et sans doute davantage inspirée par l'approche réaliste que par la construction normativiste, doit analyser comment, de manière interne, s'articulent les règles constituant l'ordre juridique de l'institution, et tout particulièrement l'État. Cette analyse est indispensable à la compréhension du droit, chacun en est d'accord, mais pour l'analyse institutionnelle, cet élément est insuffisant parce qu'il est limité au point de vue interne à l'ordre juridique, et qu'il évacue donc toute dimension sociale ;

- le niveau de la politique du droit. Ce troisième niveau établit la connexion entre le point de vue externe/ social, et le point de vue interne/ positiviste pour montrer comment, dans le milieu social, l'État se constitue en tant que système juridique spécifique, par des règles de droit qui ont vocation à régir ce milieu social, c'est-à-dire qui ont vocation à intégrer en donnant statut de droit ce qui est extérieur. II s'agit bien du niveau politique du droit : il convient de montrer la dimension politique constituant l'État, et pas simplement d'étudier l'inspiration politique ou partisane de tel ou tel texte de loi.

Le projet est vaste. II est tout autant prometteur. On se gardera de garantir toutefois que toutes les promesses pourront être tenues, parce que la force tout autant que la faiblesse de l'analyse institutionnelle ici tient à ce qu'elle est à la fois l'objet et le moyen de l'étude. En cherchant sur l'institution : le droit, on taille l'outil de la recherche: I'analyse institutionnelle. Et on doit avouer que I'on ne sait donc pas a priori et totalement où l'on va lorsque l'on s'engage dans cette analyse. Croire alors que l'analyse institutionnelle va permettre au chercheur de tout comprendre serait faire preuve d'une naïveté impardonnable. En revanche, il est tout aussi indéniable que cette analyse permet de développer sur le droit un autre point de vue, qui révèle des choses que les analyses classiques ne saisissent pas, même si, à l'évidence, il existe d'autres types d'analyse qui peuvent également révéler des éléments intéressants. Et il nous semble que chercher cet autre point de vue, essayer de saisir ce qui est caché, peut correspondre à une approche critique du droit : à une approche scientifique. 


\section{Anderson $\mathrm{P}$.}

1978, Sur Gramsci, Maspero, Paris.

\section{Beaud 0 .}

1993, « Préface » in Carl

Schmitt, La Théorie de la

Constitution, Paris, PUF.

Bobbio N.

1971, « Nouvelles réflexions sur les normes primaires et secondaires », in C. Perelman, La règle de droit, Bruxelles, Bruylant, p. 104122.

Capitant R. 1982, Écrits constitutionnels, Paris, CNRS.

Carré de Malberg R. 1962, Contribution à la théorie générale de l'État, Paris, CNRS.

Chevallier J. 1981, «L'analyse institutionnelle » in CURAPP, L'institution, Paris, PUF.

1986, Science administrative, Paris, PUF.

Chevallier J. et Loschak D. 1978, Science administrative, Paris, LGDJ .

Deleuze G. 1955, Instincts et institutions, Paris, Hachette.

Deleuze G. et Guattari F. 1972, L'anti-Oedipe, Paris, éd. de Minuit.

Despax M. 1957, L'entreprise et le droit, Paris, LGDJ .
Doat M.

1994, Recherches sur la notion de collectivité locale en droit administratif français, Thèse droit public, Université des sciences sociales, Toulouse (à paraître LGDJ , 1995).

Dubouchet P. 1993, « Pour une théorie normative de l'institution », Revue de la recherche juridique. Droit prospectif, $n^{\circ} 3$, p. $739 \mathrm{~s}$.

Durand P. et al. 1956, Traité de droit du travail, Paris, Dalloz.

Durkheim E. 1993, Les règles de la méthode sociologique, Paris, PUF.

Duverger $M$. 1955, Institutions politiques et droit constitutionnel, $\mathrm{Pa}$ ris, PUF.

Espagno D.

1991, Recherches sur la notion de service public, Mémoire de DEA de droit public, Toulouse, Université des sciences sociales.

Gounot E. 1912, Le principe de l'autonomie de la volonté en droit privé : Contribution à l'étude critique de l'individualisme juridique, Paris, Rousseau.

Habermas J .

1987, Théorie de l'agir communicationnel, Paris, Fayard. 
E. Millard

Hauriou et la théorie de l'institution

\section{Hauriou M.}

1896, La science sociale traditionnelle, Paris, Larose.

1899, Leçons sur le mouvement social, Paris, Larose.

1900, Note sous TC, 9/12/1899, Association syndicale du canal de Gignac, S. 1900.3.49.

1906, «L'institution et le droit statutaire », Recueil de législation, p. 134-182.

1910, Principes de droit public, Paris, Sirey.

1925, «La théorie de l'institution et de la fondation. Essai de vitalisme social », in Aux sources du droit : le pouvoir, l'ordre et la liberté, Cahiers de la Nouvelle J ournée, $n^{\circ} 23$ (réimprimé Université de Caen, 1990).

1929, Précis de droit constitutionnel, $2^{\mathrm{e}}$ éd., Paris, Sirey (réimprimé CNRS 1965).

Hecquard-Théron $M$.

1977, Essai sur la notion de réglementation, Paris, LGDJ.

Hegel G. W. F.

1940, Principes de la philosophie du droit, Paris, Gallimard.

Hess R. et Savoye A. 1993, L'analyse institutionnelle, Paris, PUF (coll. «Que sais-je »).

Lacan J. 1980, De la psychose paranoïaque dans ses rapports avec la personnalité, Paris, Seuil.
Langrod G. et al.

1966, Traité de science administrative, Paris, Mouton.

Linditch $F$.

1991, Recherches sur la personnalité morale en droit administratif, Thèse droit public, Toulouse, Université des sciences sociales (à paraître LGDJ , 1995).

Lourau R. 1970, L'analyse institutionnelle, Paris, éd. de Minuit.

MacCormick $\mathrm{N}$. et Weinberger $\mathrm{O}$.

1992, Pour une théorie institutionnelle du droit. Nouvelles approches du positivisme juridique, Paris, LGDJ .

Marty G. 1969, « La théorie de I'institution », in La pensée du doyen Hauriou et son influence, Paris, Pédone.

Mauss $M$.

1901, La grande Encyclopédie française, $\mathrm{V}^{\circ}$ Sociologie.

Mazères J .-A .

1990, «Les collectivités locales et la représentation, Essai de problématique élémentaire », Revue du droit public et de la science politique, p. $607 \mathrm{~s}$.

1994a, Théories institutionnelles de la connaissance juridique, Cours de DEA de droit public, Toulouse, Université des sciences sociales.

1994b, Marché et Nation : Essai d'approche juridique, Toulouse, Université des sciences sociales. 
Millard E.

1987, Genèse des doctrines de dynamique sociale de Marx et du doyen Hauriou, Mémoire de DEA de sciences politiques, Toulouse, Université des sciences sociales.

1994, Famille et droit public, Thèse droit public, Lyon, Université Jean-Moulin (à paraître LGDJ , 1995).

Mourgeon J.

1967, La répression administrative, Paris, LGDJ .

Ortiz L.

1992, Recherches sur la structure de la fonction publique territoriale, Thèse droit public, Toulouse, Université des sciences sociales (à paraître LGDJ , 1995).

Oury F. et Vasquez A. 1967, Vers une pédagogie institutionnelle, Paris, Maspero.

Renard G. 1930, La théorie de l'institution. Essai d'ontologie juridique, Paris, Sirey.

1933, «Qu'est-ce que le droit constitutionnel ? Le droit constitutionnel et la théorie de l'institution », in Mélanges Carré de Malberg, Paris, Sirey, p. 491 s.

Rivero J.

1956, «Hauriou et

l'avènement de la notion de service public », in Mélanges Mestre, Paris, Sirey.

\section{Romano S.}

1975, L'ordre juridique, Paris, Dalloz.
Roujou de Boubée G.

1961, Essai sur l'acte juridique collectif, Paris, LGDJ .

Sartre J.-P.

1960, Critique de la raison dialectique, tome 1 : Théorie des ensembles pratiques, $\mathrm{Pa}$ ris, Gallimard.

Scelle G.

1948, Droit international public, Paris, Domat.

Searle J.

1972, Les actes de langage. Essai de philosophie du langage, Paris, Hermann.

Ségur $P$.

1993, Recherches sur les relations du pouvoir politique et du temps, Thèse, Toulouse, Université des sciences sociales.

Sfez L.

1966, Essai sur la contribution du doyen Hauriou au droit administratif français, Paris, LGDJ .

Tanguy $\mathrm{Y}$. 1991, «L'institution dans I'œuvre de Maurice Hauriou. Actualité d'une doctrine », Revue du droit public et de la science politique, p. 6179.

Tosquelles $\mathrm{F}$. 1969, Psychothérapie institutionnelle, Paris.

Vedel G. 1954, « Les bases constitutionnelles du droit administratif », Etudes et documents du Conseil d'État, n8, p. 2153. 